\title{
Editorial
}

\section{Can Multidrug Resistance in Tuberculosis be Curbed?}

Tuberculosis (TB) is an ancient disease, perhaps 3 million years old. The term Tuberculosis was coined way back in 1834 by Johann Schonlein. It was a dreaded disease as evident from its earlier names like 'phthisis' in ancient Greece, 'tabes' in ancient Rome, 'schachepheth' in ancient Hebrew, the 'white plague', 'white death', 'consumption', 'lung fever', 'galloping fever', 'graveyard cough' and 'Captain of men of death'. TB of the neck and lymph nodes was called scofula, believed to be different from pulmonary TB.

Currently, on the basis of treatment, TB is categorized into drug-susceptible, drug-resistant, multidrug resistant (MDR) and extensively drug-resistant (XDR). MDR-TB is disease that does not respond to at least isoniazid and rifampicin, the two most powerful anti-TB drugs. XDR-TB is a form of MDR-TB with additional resistance to more anti-TB drugs that therefore responds to even fewer available medicines. XDR-TB is accurately defined as in vitro resistance to first-line anti-TB drugs isoniazid and rifampicin plus any second-line oral drugs: fluoroquinolone and at least one of the second-line injectable drugs: amikacin, capreomycin or kanamycin. A label of Latent TB is given when a person has the TB bacteria within his/her body in very minute quantities that are kept under control by the body's immune system and do not cause any symptoms and are not infectious. It is estimated that 40-50 $\%$ of the Indian population has Latent TB.

Robert Koch isolated the tubercle bacillus, presented his finding to the Society of Physiology in Berlin on 24 March 1882 and also contributed to the elucidation of the infectious etiology of TB (1). A century later, March 24 was designated by the World Health Organization (WHO) as the World TB Day. In 1905, Koch won the Nobel Prize for Medicine and Physiology (2). In subsequent decades, the Pirquet and Mantoux tuberculin skin tests, Albert Calmette and Camille Guérin BCG vaccine, Selman Waksman streptomycin and other anti-TB drugs were developed (2). Dr. Koch's discovery was the most important step taken towards the control and elimination of this deadly disease. Having the capacity to spread rapidly being an airborne infectious disease and developing resistance to the commonly used medications, it is high time to identify the weak areas that need attention to curb the disease.

TB continues to be a major public health problem. A combined strategy, based on improving drug treatment, diagnosis and prevention modalities, is necessary in order to eradicate Mycobacterium tuberculosis by the year 2030, as committed by the WHO (3). Elimination as defined by the WHO is less than one case of TB for a population of a million people.

India has the highest number of deaths from TB in the world since the bacterial infection kills one person every minute (4). The latest report from WHO states that $33 \%$ of deaths worldwide related to TB excluding HIV occurred in India in 2017 (5). As per the Global TB report 2017, the estimated incidence of TB cases in India was approximately 28,00,000 accounting for about a quarter of the world's TB cases (5). MDR-TB is on the rise across India, with an incidence of 1,47,000 cases (5). The two main 
reasons why MDR-TB continues to emerge and spread are mismanagement of TB treatment and person-to-person transmission.

The reason TB found easy access to spread in India was the highly populous areas living in closed crowded locations with poor immunity added to the lack of awareness and personal hygiene. The disease is more common in certain states of India. Earlier, the poor were affected more and did not mind getting admitted in TB sanatoriums that was beneficial to them as they were properly looked after, got nutritious food and timely medications. Slowly the disease has now affected even the urban population as the rural people have migrated to towns and live in close contacts with the urban population as house care takers or workers in restaurants, factories, government offices or public and private funded hospitals. Another angle yet to be explored is the presence of TB in animals like cows, cats and dogs (6). There are innumerable stray animals in India who if infected can also contribute to spread of the disease, albeit of other etiologic species of Mycobateria.

The liberal availability of over-the-counter (OTC) drugs like Ciprofloxacin and liberal use of injectable Amikacin which are also second-line anti-TB drugs has led to development of resistance. Poor resources and lack of awareness of the communicable nature have led to spread of the disease. The patients, in order to save time and avoid travel, seek intermittent help from private practitioners and thus are not in close supervision or monitoring for compliance and response. Other reasons to seek private treatment include lack of awareness of disease symptoms, poor knowledge of free services available through the national public program, desire for confidentiality and personalized care. Lack of knowledge is a big factor that contributes to MDR-TB. The patients are non-compliant; after few weeks of treatment, they feel that have got well and leave the prescribed course of medicines.

In India, there are no guidelines to protect the other patients, staff and visitors once an infected case is admitted in Hospital till date. When I was trained as PG, each year two residents out of 4 in the unit used to get infected with the disease despite occasional use of the mask. However, in those days we used to routinely wash the loaded under-water seal chest tube drain bags and fill it with clean saline for reuse. Fortunately, the public hospitals have now been provided with sufficient funds for the chest bags. Patient should not spit on the ground as the infected sputum gets mixed with the dust particles and spreads the infection. Those infected should ideally either run their sputum into a drain, bury their collected sputum or burn it to prevent spread of infection. However, such instructions are not passed to majority of the patients by the doctors, who themselves might be misinformed or lack desire to inform. During General Surgery training, I was fortunate to work under a cardiothoracic surgeon and assisted hundreds of cases of chest TB surgeries including pneumonectomy, lobectomy, thoracoplasty and pericardectomy (7). It used to be a heroic surgery and thanks to the discovery of highly efficacious antiTB drugs and designing of evidence-based anti-TB regimens, such an expertise is limited as the surgery for pulmonary TB is no longer in vogue. Currently, video-assisted thoracic surgeries or decortications are done with occasional lobectomy for very sick patients. The surgical expertise is also fading away with minimal invasive techniques that have the disadvantages of lack of palpation of the unhealthy lobe leading to incomplete resection.

The referral system also delays timely treatment. The super specialists forget the training they got during undergraduation or postgraduation and prefer to refer the patients to their colleagues for requesting investigations and writing the doses of the anti-TB drugs. Initiation of different protocols of anti-TB therapy in various hospitals may also lead to conversion of TB cases to MDR-TB or XDR-TB. 
The large scale implementation of the Indian government's Revised National TB Control Program (RNTCP/ RNTCP I) was started in 1997. The entire nation was covered by the RNTCP. In March 2006, it was re-designated as RNTCP II that was designed to consolidate the gains achieved in RNTCP I, and to initiate services to address TB/HIV, MDR-TB and to extend RNTCP to the Private Sector. The RNTCP is responsible for carrying out the Government of India Five-year TB National Strategic Plan (NSP). Under the RNTCP both the diagnosis and treatment of TB were free. There is, theoretically, no waiting period for patients seeking treatment and TB drugs.

Contrary to WHO target of eliminating TB by 2030, the Government of India is calling for the elimination of TB by 2025, and there is a new NSP 2017-2025 with emphasis on reaching patients seeking care from private providers, building on the work already done with the new RNTCP guidelines. The RNTCP will also be helping private practitioners and hospitals to provide quality care and treatment, rather than encouraging the private providers to send their patients to get care from the RNTCP. The NSP plans to provide incentives to private providers for following the standard protocols for diagnosis and treatment as well as for notifying the government of cases. Also patients referred to the government will receive a cash transfer to compensate them for the direct and indirect costs of undergoing treatment and as an incentive to complete the course of treatment.

New WHO recommendations aim to speed-up detection and improve treatment outcomes for MDR-TB through use of a novel rapid diagnostic test and a shorter, cheaper treatment regimen. New technologies like whole genome sequencing help public health professionals see the patterns of TB transmission. The Genexpert test is a molecular test for TB (MTB) which diagnose TB by detecting the presence of TB bacteria DNA, as well as testing for resistance to the drug rifampicin. In December 2010, WHO endorsed the Genexpert technology and released a recommendation and guidance for countries to incorporate the new test into their anti-TB programs (8). In India, this test is known as the Cartridge Based- Nucleic Acid Amplification (CB-NAAT) Test. It uses a sputum sample and can give a result in less than 2 hours. It can also detect the genetic mutations associated with resistance to rifampicin.

In 2017 the Xpert Ultra assay was launched and found to be non-inferior to the standard Xpert MTB assay for the diagnosis of TB and the detection of rifampicin resistance. A new device called the GeneXpert Omni is currently under development. It is intended for point-of- care (POC) testing for TB and rifampicin resistance, using the same cartridges as those used in the current Genexpert machine.

Two new medicines have been introduced in the government-run hospitals with restricted, but available to those who require them most: bedaquiline and delamanid recommended by WHO for patients with XDR-TB who have documented evidence of resistance to any fluoroquinolones. Patients with TB are tested for HIV and patients with HIV are tested for TB. Drug treatment is moving from intermittent therapy to daily fixed-dose combinations (FDCs). Among repurposed drugs for the treatment of MDRTB are clofazimine, linezolid, meropenem/clavulanate and ertapenem. Other regimens include high dose isoniazid (16-18mg/kg), pretomanid, moxifloxacin and pyrazinamide, addition of ethambutol if not added earlier, Aminoglycosides (kanamycin, and amikacin) and Polypeptides antibiotics (capreomycin and viomycin).

To conclude, to curb and prevent MDR-TB and XDR-TB, we need to identify contacts who could have contracted TB, promote awareness, improve environmental hygiene, encourage rapid diagnosis and appropriate treatment, assess response to treatment, assure compliance, identify and screen 
immunodeficient people like those infected with HIV for presence of TB. Social and public health interventions need to supplement the efforts of the government to reach the desired goal of TB eradication.

\section{Shilpa Sharma}

Associate Professor, Department of Pediatric Surgery, All India Institute of Medical Sciences, New Delhi. New Delhi-110029. Email: drshilpas@gmail.com

\section{References}

1. Gradmann C (2001). Robert Koch and the pressures of scientific research: tuberculosis and tuberculin. Medical History 45: 1-32.

2. Barberis I, Bragazzi NL, Galluzzo L, Martini M (2017). The history of tuberculosis: from the first historical records to the isolation of Koch's bacillus. J Prev Med Hyg 58: E9-E12.

3. Agoramoorthy G (2017). India needs to refine a strategy to tackle the tuberculosis epidemic. Lancet Infectious diseases 17: 23-24.

4. Ministry of Health \& Family Welfare (2018). Government of India. Central Tuberculosis Division. Directorate General of Health Services Annual report 2018.

https://tbcindia.gov.in/showfile.php?lid=3314 Last accessed March, 2018.

5. Dye C, Williams BG (2008). Eliminating human tuberculosis in the twenty-first century. $J R$ Soc Interface 5: 653-662.

6. Parsons SD, Warren RM, OttenhoffTH, Gey van Pittius NC, van Helden PD (2012). Detection of Mycobacterium tuberculosis infection in dogs in a high-risk setting. Res Vet Sci 92: 414-419.

7. Narula IMS (1996). Professor and Head, Department of Surgery, RNT Medical College, Udaipur, Rajasthan.

8. WHO meeting report of a technical expert consultation (2017): Non-inferiority analysis of Xpert MTB/RIF Ultra compared to Xpert MTB/RIF", Geneva, World Health Organisation; 2017, www.who.int/tb/areas-of-work/laboratory/ last accessed April 2018. 